\title{
Bifid Shape Is Intrinsic to Bifidobacterium adolescentis
}

\author{
Dhanashree ${ }^{1}$, Sharika Rajashekharan², Balamurugan Krishnaswamy² and \\ Rajagopal Kammara ${ }^{1 *}$
}

${ }^{1}$ Department of Protein Chemistry and Technology, Council of Scientific and Industrial Research - Central Food Technological Research Institute, Mysore, India, ${ }^{2}$ Department of Biotechnology, Alagappa Univeristy, Karaikudi, India

Although the genus Bifidobacterium was originally named for its bifid morphology, not all bifidobacterial species have a similar structure, and very few of them adopt a bifid shape under stress conditions. The exposure of respective bifidobacterial species to various conditions, such as different $\mathrm{pH}$, temperatures, medium components, in vivo growth in Caenorhabditis elegans, and subculture, did not affect their diverse morphologies. Extensive scanning electron microscopy studies suggested that the bifid shape of $B$. adolescentis are maintained irrespective of the conditions. Hence, we conclude that the bifid morphology is intrinsic to $B$. adolescentis. Most bifidobacterial species are anaerobic and rod-shaped, whereas, after the first generation, they become

OPEN ACCESS

Edited by:

Andrea Gomez-Zavaglia,

Center for Research and

Development in Food Cryotechnology

(CONICET), Argentina

Reviewed by: Atte Von Wright,

University of Eastern Finland, Finland Alberto Finamore,

Food and Nutrition Research Center

(CREA), Italy

*Correspondence:

Rajagopal Kammara krgopal22@ rediffmail.com

Specialty section:

This article was submitted to Food Microbiology,

a section of the journal

Frontiers in Microbiology

Received: 12 December 2016 Accepted: 08 March 2017 Published: 21 March 2017

Citation:

Dhanashree, Rajashekharan S, Krishnaswamy $B$ and Kammara $R$ (2017) Bifid Shape Is Intrinsic

to Bifidobacterium adolescentis.

Front. Microbiol. 8:478.

doi: 10.3389/fmicb.2017.00478 microaerophilic or aerophilic. $\mathrm{CaCl}_{2}$ (treatment of $B$. animalis) signaling triggered a change from the rod shape to the bifid shape and vice versa in $B$. adolescentis.

Keywords: bifid, polymorphic, Bifidobacterium, intrinsic, aerophilic, microaerophilic

\section{INTRODUCTION}

The genus Bifidobacterium is so designated because in the reference species, one end was a rocketshaped and the other, longitudinally split (Tissier, 1900). Various bifidobacterial species have been classified based on the nucleotide sequence encoding the 16S rRNA, Hsp60, and xylulose-5phosphate (XFP; also called fructose-6-phosphate phosphoketolase/F6PPK). However, the lack of cutting-edge research into the taxonomy of the genus Bifidobacterium has meant that these species have been retained in Bifidobacterium, regardless of morphological differences. Because very few bifidobacterial strains have a bifid shape, even though some are polymorphic both rod-shaped and bifid-shaped species are retained in the single genus Bifidobacterium.

Investigators in the field, such as Weiss (1933), Blaurock (1937), Boventer (1938), Weiss and Rettger (1938), and Tomarelli et al. (1949a,b), have stressed the demand to develop optimal media for bifidobacterial s pecies. The reflection of variations in the bifid structures of Bifidobacterium and Lactobacillus acidophilus was confirmed by Herter (1907), Bliuhdorn (1910), Kendall and Haner (1924). Since then, some have suggested that Bifidobacterium and Lactobacillus are two different genera (Sundman et al., 1959). Exposure to stress conditions causes the latter to adopt pleomorphic forms, including the bifid form. These observations were confirmed by Rodella (1908), Cruickshank (1925), Roos (1927), and Synder et al. (1967) who reported that the alternative forms are uncommon and give an illusion of branching. Norris et al. (1950) confirmed that stress conditions induce the polymorphic forms of Bifidobacterium. However, the mechanisms

Abbreviations: bp, base pair; F6PPK, fructose-6-phosphate phosphoketolase; Ocins, antimicrobial peptides/proteins of Lactobacillus, Bifidobacteria, and Enterococcus spp.; OD, optical density; xfp, xylulose-5-phosphate/fructose-6-phosphate phosphoketolase. 
underlying the bifid conformation are unknown, and there has been no account of this polymorphic/bifid morphology. Therefore, in this study, we investigated the bifid nature of Bifidobacterium. Studies of polymorphic bacterial morphologies by Moro (1905) and Tissier (1905) demonstrated that bifidshaped organisms are present in the fecal material of breastfed infants. Populations of these organisms (bifid form) in the intestine represent the disease-free condition and are physiological significance. However, the cellular elements and physical parameters responsible for the upkeep of the bifid form in vivo and in vitro are yet to be determined.

According to available reports, there are about 63 different Bifidobacterium species. The nomenclature and taxonomy of these microbes are incomplete. Hence, it is critical to understand the diverse nature of Bifidobacterium and to determine the characteristic morphologies of all the Bifidobacterium. It is essential to determine whether this polymorphism is intrinsic to a particular species of Bifidobacterium. Weiss and Rettger (1938), reported that these bacteria lose their bifid structure and change to the rod-shaped form in the subculture. Moreover, before subculture, they are strictly anaerobic, whereas after subculture, they are aerobic. The mechanisms of all these phenomena are yet obscure. In this study, we examine the polymorphic morphologies of the Bifidobacterium, and conclude that the bifid morphology is an intrinsic characteristic of B. adolescentis, whereas (very few) other species, including $B$. animalis, display polymorphic behavior only under stress conditions. However, all the other bifidobacterial species maintain their rod shape, regardless of the culture conditions, and rarely adopt the bifid shape.

In the Homma et al. (1960) showed that univalent cations induce this unique phenomenon. Kojima et al. (1970) confirmed the role of monovalent cations, including sodium chloride, sodium sulfate, sodium nitrate, tribasic sodium phosphate, and sodium acetate, in the induction of the bifid form. Later, the roles of chlorides of $\mathrm{Ca}^{++}, \mathrm{Ba}^{++}, \mathrm{Mg}^{++}, \mathrm{Mn}^{++}$, and $\mathrm{Zn}^{++}$were shown to play no role in this pleomorphism (Kojima et al., 1970). In the $1960 \mathrm{~s}$, glucose and sucrose were also proven to positively affect this polymorphism (Kojima et al., 1968).

Tissier (1899), L. bifidus was the only organism that was exhaustively dissected for its morphological variability. However, this work was incomplete because many bifidobacterial species are recognized today and many decades-old comparative morphological studies have not survived. In response to the original studies, the name 'Bifidobacterium' was suggested, but was considered equivocal, even then. In those days, fewer Bifidobacterium species were available, and L. bifidus may have been the only recognized bifidobacterial species. In 1950, 1968, and 1970, L. bifidus was the only strain in which this polymorphism was investigated. Kojima et al. (1970) demonstrated the role of $\mathrm{CaCl}_{2}$ in inhibiting various bacterial morphological forms. The comparative study (by Kojima et al., 1970) of cell wall composition of the bifid and bacilloid forms reported are clearly shown the restriction of amino acid phenylalanine and methionine to bifid form and the presence of high content of the glucose compared to bacilloid form. Surprisingly, larger peptidoglycans were found in the bacilliform, whereas the average-sized peptidoglycans occur in the bifid form. These observations also support the contention that 'Bifidobacterium' is a misnomer. Not all Bifidobacterium shows the bifid morphology because it is not an intrinsic characteristic, and may be restricted to very few members of the genus.

To examine the different morphologies of the species included in the genus Bifidobacterium, we selected several bifidobacterial groups constructed by Bottacini et al. (2014), each selected species corresponds to a particular group and subjected them to different stress conditions. The different Bifidobacterial groups were fed to the model system, Caenorhabditis elegans to assess their morphological changes during in vivo stress conditions and was characterized and analyzed through scanning electron microscopy (SEM). In this manuscript, we report our findings on the varied morphologies of the Bifidobacterium, detected with SEM and compound microscopy.

\section{MATERIALS AND METHODS}

Organisms: Bifidobacterium species, including B. animalis, $B$. longum, and B. adolescentis, etc., were obtained from the German collection of Microorganisms and Cell Cultures (Inhoffenstraße 7B, 38124 Braunschweig, Germany). For details, see Table 1. C. elegans and OP 50 Escherichia coli was a kind gift of Dr. Balamurugan.

\section{Media and Culture Conditions}

Commercially available media, such as MRS (Oxoid, Milano, Italy) and Bifidobacterial broth (Hi-Media Laboratories, A-516, Swastik Disha Business Park, via Vadhani Industrial Estate, L.B.S. Marg, Mumbai-400 086, India) and agar medium, were used to grow the different Bifidobacterial species. The first generation of the Bifidobacteria was cultured in MRS broth (and also in Bifidobacterial broth when appropriate) at $37^{\circ} \mathrm{C}$ (without shaking) in an anaerobic chamber for $36 \mathrm{~h}$. They were sub-cultured and grown in aerobic condition in the second generation.

\section{Growing, Harvesting, and Growth Curve Studies}

Bifidobacterial strains, B. animalis, B. longum, and B. adolescentis, were grown at different temperatures: room temperature, 30, 37, and $42^{\circ} \mathrm{C}$. The bifidobacterial strains were streaked onto the MRS agar plate to produce single isolated colonies. The streaked plates were incubated at $37^{\circ} \mathrm{C}$ (for $36 \mathrm{~h}$ ) under anaerobic conditions. A single colony was isolated, used to inoculate $10 \mathrm{ml}$ of fresh MRS medium, and grown in an anaerobic growth chamber. An aliquot (1\%) of each inoculum was subculture in $15 \mathrm{ml}$ of fresh MRS medium to construct growth curves. The bacterial samples were collected every $6 \mathrm{~h}$ and the $\mathrm{OD}_{600}$ measured. All the experiments were performed in triplicates.

\section{Growth, Maturation, and Harvesting of C. elegans}

Wild-type C. elegans were obtained from CGC and maintained on NGM at $20^{\circ} \mathrm{C}$. The standard E. coli OP50 strain was used as a 
TABLE 1 | Strains used in this study.

\begin{tabular}{ll}
\hline Bacterial strain \\
\hline 1 & B. animalis subspp. lactis \\
2 & B. thermoacidophilum subspp. thermoacidophilum \\
3 & B. adolescentis \\
4 & B. longum subspp. infantis \\
5 & B. longum subspp. longum \\
6 & B. asteroides \\
7 & B. animalis \\
8 & B. breve \\
9 & B. indicum \\
10 & E. coli OP50
\end{tabular}

Strain no.

DSMZ 10140

DSMZ 15837

DSMZ 20083

DSMZ 20088

DSMZ 20219

DSMZ 20089

DSMZ 20105

DSMZ 20213

DSMZ 20214
Reference

Meile et al., 1997

Dong et al., 2000

Reuter, 1963

Reuter, 1963

Reuter, 1963

Scardovi and Trovatelli, 1969

Mitsuoka, 1969

Reuter, 1963

Scardovi and Trovatelli, 1969

A gift from Prof. Balamurugan

$B$, Refers to Bifidobacterium.

food source. Age synchronized young adult worms were used in all experiments. Synchronization was obtained through bleaching of gravid hermaphrodites to obtain eggs that were plated onto seeded plates. The young adult nematodes were harvested from NGM plates by using M9 buffer and used for experiments. Was grown on NGM agar plates and fed internationally accepted feed (the standard E. coli OP50 strain), as described previously (Ward, 1973). Standard techniques (Hope, 1999; Stiernagle, 1999) for the maintenance and growth of nematodes on NGM were used.

\section{Bacterial Strains and Growth Conditions for C. elegans Feeding}

Escherichia coli $\mathrm{OP} 50$ was grown overnight at $37^{\circ} \mathrm{C}$ at $200 \mathrm{rpm}$ in Luria-Bertani medium. The bacterial lawns used for the C. elegans studies were prepared by spreading $10 \mu \mathrm{l}$ of the bacterial strain onto nematode growth media (NGM) agar $(0.25 \%$ peptone) in $3.5-\mathrm{cm}$ plates. The plates were incubated overnight in an incubator at $37^{\circ} \mathrm{C}$. They were allowed to equilibrate to room temperature before they were seeded with C. elegans and incubated at $20^{\circ} \mathrm{C}$ throughout the experiment.

All the bifidobacterial strains cited in the Table 1 (except E. coli) were grown as shown above. Bacterial lawns were made by spreading $10 \mu \mathrm{l}$ of the bifidobacterial strains onto MRS agar in $3.5-\mathrm{cm}$ plates. A similar procedure to that described above was followed, but $C$. elegans was fed different Bifidobacterial (B. longum and B. adolescentis) strains rather than E. coli OP50. $B$. longum and $B$. adolescentis were considered further for these studies as it is preferred by the C. elegans. The second generation of this bacteria is microaerophilic therefore they can grow in aerobic condition, where C. elegans also grows.

\section{Chemotaxis Assay}

To determine whether the Bifidobacterium and their products (ocins) are non-toxic and safe for C. elegans, several experiments were performed (Brenner, 1974). The procedure follows as below. A Petri plate was divided into four parts, two opposite and one adjacent part labeled "Test" $(A, B$, and $D)$ and the remaining as "Control" (C). All the sectors (A, B, C, and D) were equidistant from one another. Part A contained B. animalis, part B B. longum, part C the control E. coli OP50, and part D B. adolescentis. Approximately, 25 wild-type $C$. elegans that had been grown on
E. coli OP50 were washed thoroughly and introduced into the center of the plate and the origin was marked with a circle to allow non-motile worms to be eliminated. The plates were incubated under optimal conditions, and analyzed the following day. All the bacteria were equidistant from the center where the worms started their journeys. The numbers of worms in each bacterial sector were scored after 12 and $24 \mathrm{~h}$ and the chemotaxis index (CI) was calculated by using the following formula.

$$
\mathrm{CI}=\frac{\text { Worms on test bacterium }- \text { Worms in E. coli OP50 }}{\text { Total number of worms }}
$$

\section{Phenotypic Observations of Bifidobacterium-Fed C. elegans and C. elegans as a Model Organism to Understand the Polymorphic Morphologies of Bifidobacterium}

Caenorhabditis elegans was used as a model organism to determine the ability of the nematode to consume Bifidobacterium and to understand the intrinsic morphology of B. adolescentis. Different Bifidobacterium (B. animalis, B. longum, and $B$. adolescentis) were grown in the appropriate medium, as described above. They were harvested, washed, and resuspended in the appropriate medium. C. elegans were fed with different ratios (1:10, 1:20, and so on) of Bifidobacterium and left for 72-96 h. They were harvested at different intervals (after 12, 24, $32 \mathrm{~h}$, and so on) without rupture, and then smashed so that the internal microbes could be observed. The worms were washed, fixed with glutaraldehyde, after a series of wash animals were crushed on the slide and analyzed with SEM. The phenotypic changes in the nematodes fed Bifidobacterium were observed microscopically. The cells of B. adolescentis-fed worms were also lysed, and the supernatants observed with SEM and the bacteria were quantified (CFU).

\section{Bifidobacterial (B. longum and B. adolescentis) Colonization of the C. elegans Digestive Tract}

The method of Garsin et al. (2001), with minor modifications, was used to estimate the approximate numbers of bacterial cells 
in the worms. Ten worms were selected, and the surface bacteria were removed by washing the worms $4-5$ times with $5 \mu \mathrm{l}$ of M9 buffer on agar plates. Each worm was transferred to a $0.5 \mathrm{ml}$ centrifuge tube containing $20 \mu \mathrm{l}$ of M9 buffer and mechanically disrupted with a Microtube pestle (Scientific Specialties, Inc., Lodi, CA, USA). The volume was then adjusted to $100 \mu \mathrm{l}$ with M9 buffer (serially dilute and spread) and the numbers of bacteria [colony-forming units (cfu)] were estimated by spreading the culture on MRS agar plates. The plates were incubated at the appropriate temperature until the colonies were observed. The same colonies were washed well with M9 buffer and immediately fixed and observed with SEM. Genomic DNA was isolated from some bacteria and subjected to $16 \mathrm{~S}$ rRNA amplification and alignment.

\section{Understanding the Bifidobacterial Morphology through SEM}

Scanning electron microscopy studies were conducted to understand the diverse morphology of Bifidobacterium. Various Bifidobacterium species were subjected to different conditions, including different media (MRS or Bifidobacterium broth) and different temperatures (in MRS at room temperature, 30, 37, or $42^{\circ} \mathrm{C}$ for SEM and Growth curve studies). They were also grown in MRS medium (for three generations also), with or without amino acids $(667 \mathrm{mg} / \mathrm{lt})$ (alanine + asparagine + glutamine + serine, or individually with alanine, asparagine, tryptophan, etc.) in the presence and absence of $\mathrm{CaCl}_{2}$ (Husain et al., 1972), and on MRS with and without salts $\left(0.2 \mathrm{M} \mathrm{Na}_{2} \mathrm{SO}_{4}, 0.2 \mathrm{M}\right.$ $\mathrm{NaCl}$, or $0.2 \mathrm{M} \mathrm{CH}_{3} \mathrm{COONa}$ ) (Kojima et al., 1968, the effect of salt concentration studies) and at different $\mathrm{pH}(5-7.5)$. The pleomorphism of Bifidobacteria was understood by growing them in vivo (in C. elegans).

\section{The Procedure Followed for SEM}

The various bifidobacterial strains were harvested, and washed twice with phosphate buffer. The pelleted bacteria were fixed in $2 \%$ glutaraldehyde and incubated at $4^{\circ} \mathrm{C} 1 \mathrm{~h} /$ overnight. The cells were then harvested and washed with a $10-100 \%$ gradient of ethanol. The cells were resuspended in 50-100 $\mu$ l of absolute alcohol. Approximately $2 \mu \mathrm{l}$ of the sample was placed on a cover slip, dessicated and examined with SEM. The images were collected at 20,000 magnifications. Only appropriate and convincing images were considered in the analysis.

\section{Genomic DNA Isolation}

The commercial GeneJET Genomic DNA Purification Kit (Fermentas, Inc., 830 Harrington Court, Burlington, ON L7N $3 \mathrm{~N} 4$, Canada) was used for genomic DNA isolation. In brief, a single isolated colony was used to inoculate $10 \mathrm{ml}$ of MRS broth and grown overnight at $37^{\circ} \mathrm{C}$ without shaking under anaerobic conditions. The following day, the cells were harvested and heat treated at $37^{\circ} \mathrm{C}$ in the presence of $20 \mu \mathrm{g}$ of lysozyme in Tris$\mathrm{HCl}$ and EDTA buffer ( $\mathrm{pH}$ 8.0). After treatment with proteinase $\mathrm{K}$, RNAse A was added and the cells were incubated for $10 \mathrm{~min}$ at room temperature. The resulting mix was centrifuged at $12,000 \mathrm{rpm}$ for $20 \mathrm{~min}$, and the supernatant was transferred to a GeneJET column. After two washes with wash buffer, the DNA was finally eluted with Tris buffer ( $\mathrm{pH} 8.0$ ).

\section{S rRNA Amplification, Sequence Analysis, and Alignment}

The genomic DNA extracted as described above was used as the template for the PCR amplification of 16S rRNA using the bifido16S universal primers 357F/926R (357F 5'-CCTACGGGAGGCAGCAG-3'; 926R 5'-CCGTCAATTCMT TTRAGT- $3^{\prime}$ ) and DyNAzyme II DNA polymerase (Thermo Scientific). The cycling parameters were: initial denaturation at $95^{\circ} \mathrm{C}$ for $5 \mathrm{~min}$, followed by $35 \mathrm{cycles}$ at $95^{\circ} \mathrm{C}$ for $30 \mathrm{~s}, 56^{\circ} \mathrm{C}$ for $45 \mathrm{~s}$, and $72^{\circ} \mathrm{C}$ for $1 \mathrm{~min}$, and a final extension at $72^{\circ} \mathrm{C}$ for $5 \mathrm{~min}$. Each reaction was analyzed on $1 \%$ agarose gel. The resulting PCR products were subjected to DNA sequencing with an ABI 310 DNA sequencer (institutional facility). Sequence similarity was determined with the National Center for Biotechnology Information BLAST tool (NCBI).

\section{RESULTS}

The analysis of the 16S rRNA nucleotide sequences, Hsp60, and F6PPK showed distinct differences between the Bifidobacterium species. From the group three species were chosen for the study as they symbolize all the Bifidobacteria species. Certainly temperature does play a major part in the growth of Bifidobacteria. All together the three species [B. animalis subsp. lactis (DSM accession no. 10140), B. adolescentis (DSM accession no. 20083), and B. longum (DSM accession number: 20219)] grew well at $37^{\circ} \mathrm{C}$ reaching an $\mathrm{OD}_{600}$ of $3.0-5.0$ after $66 \mathrm{~h}$ of culturing (Figure 1B). When the temperatures were reduced to $30^{\circ} \mathrm{C}$ the growth rates were drastically reduced (Figure $1 \mathrm{~A}$ ). The whole of the Bifidobacteria species were unable to come to an $\mathrm{OD}_{600}$ of 3.0 even after $72 \mathrm{~h}$ of growth. Nevertheless, considerable growth was observed for $B$. adolescentis. The remaining two Bifidobacteria grew very slowly, and an extended lag phase was discovered. When the growth curves were observed at an increased temperature of $42^{\circ} \mathrm{C}$ (Figure 1C) an outcome similar to that discovered at lower temperatures was observed (Figure 1A). Nevertheless, the cells did not obtain an $\mathrm{OD}_{600}$ above 3.0 , even after $72 \mathrm{~h}$ of growth. These results indicate that $37^{\circ} \mathrm{C}$ may be an optimal temperature for the growth of these bacterial strains (Figure 1B). The growth curve studies further emphasize that $B$. animalis subsp. lactis is very slow growing (obtained an $\mathrm{OD}_{600}$ of 2.0-2.5), and it maintained this growth rate at different temperatures $\left(30-42^{\circ} \mathrm{C}\right)$. B. longum showed the greatest growth rates at $37^{\circ} \mathrm{C}$, and its growth rate was greater than either of the other strains. Strikingly, B. adolescentis grew faster at $37^{\circ} \mathrm{C}$, reaching an $\mathrm{OD}_{600}$ of $3.0 \mathrm{in} 30 \mathrm{~h}$, a task which takes $48 \mathrm{~h}$ at $30^{\circ} \mathrm{C}$ and $72 \mathrm{~h}$ at $42^{\circ} \mathrm{C}$.

Three different Bifidobacteria such as Bifidobacterium adolescentis, B. animalis, and B. longum was grown under different concentrations of oxygen and their growth curves analyzed. The results clearly demonstrated that as the concentration of oxygen in the system increased, the multiplication rate of the bacteria decreased and vice versa 

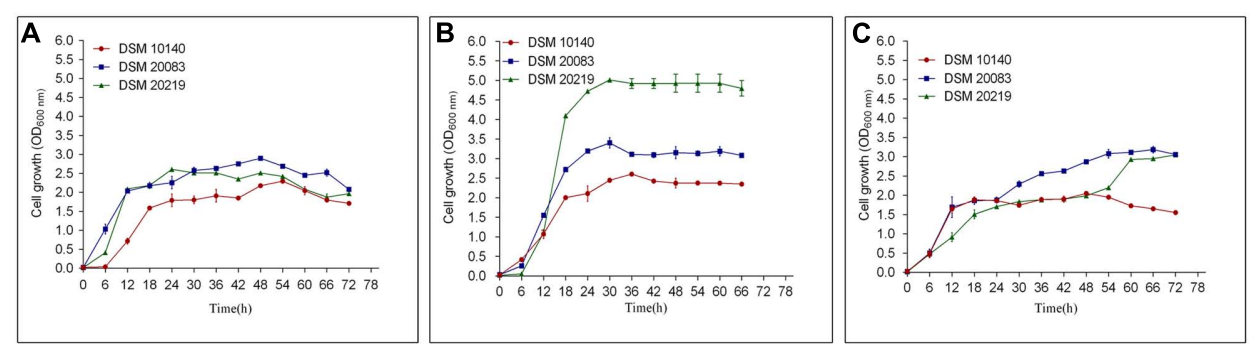

FIGURE 1 | Growth curves of DSM 10140 (B. animalis subspp. lactis), DSM 20083 (B. adolescentis), DSM 20219 (B. longum) at different temperatures. (A) $30^{\circ} \mathrm{C}$, (B) $37^{\circ} \mathrm{C}$, (C) $42^{\circ} \mathrm{C}$
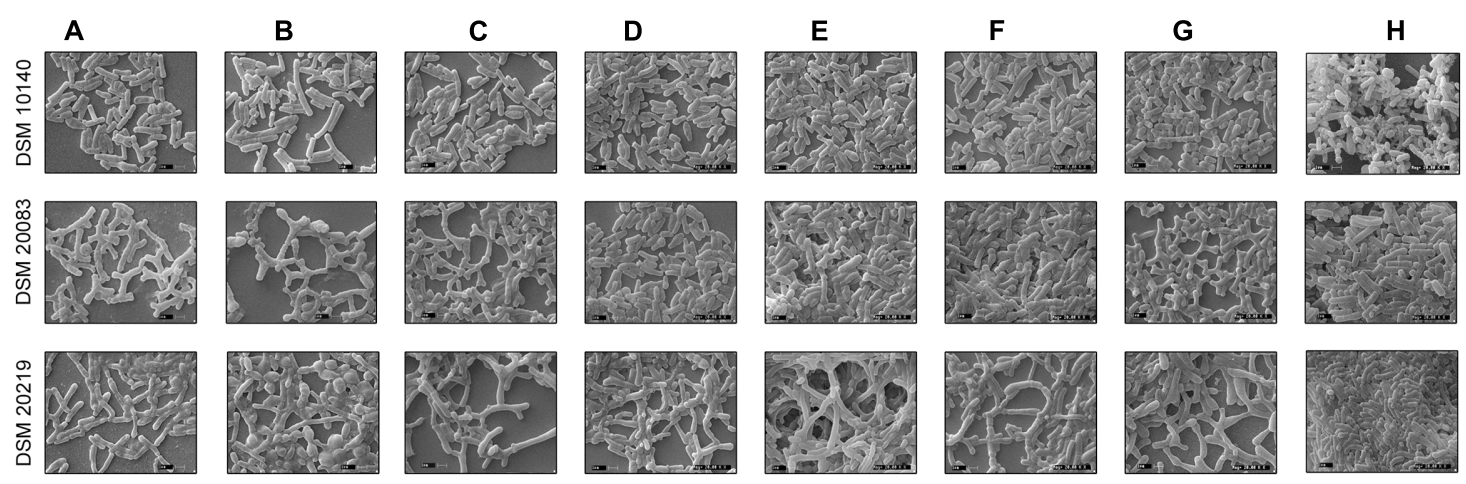

FIGURE 2 | Scanning electron microscopy (SEM) of strains DSM 10140 (B. animalis subspp. lactis), DSM 20083 (B. adolescentis), DSM 20219 (B. longum) with different conditions like (A) $37^{\circ} \mathrm{C}$, (B) $42^{\circ} \mathrm{C}$, (C) media with alanine, aspartate, glutamate, serine, (D) media with alanine, (E) media with tryptophan, (F) media with asparagine, (G) media with $0.2 \mathrm{M}$ sodium acetate, $\mathbf{( H )}$ media with $0.25 \mathrm{M}$ sodium chloride.

(data not shown). The different Bifidobacterial species displayed different resistance and susceptibility to oxygen (in the first generation). In the second generation, all the species grew aerobically, irrespective of the presence (of different concentrations) of oxygen. An oximetric study also showed that each Bifidobacterium species required different amounts of oxygen for growth, especially in the first generation. This further confirms that Bifidobacteria does show intolerance in the IInd generation, equally it is sensitive in the first generation (Beerens et al., 2000; Kawasaki et al., 2006; Ruiz et al., 2011).

To investigate the various morphologies of the Bifidobacterial species, they were subjected to various conditions and analyzed with SEM (Figures 2A-H). The experiments were performed at different temperatures $\left(37,42^{\circ} \mathrm{C}\right.$; Figures $\left.2 \mathrm{~A}, \mathbf{B}\right)$, in different media (Supplementary Information), supplemented with an amino acid mixture containing alanine, aspartate, glutamate, and serine (Figure 2C), and also individually supplemented with alanine (Figure 2D), asparagine (Figure 2F), tryptophan (Figure 2E), or salts such as $0.2 \mathrm{M} \mathrm{CH}_{3} \mathrm{COONa}$ (Figure 2G) and $0.25 \mathrm{M} \mathrm{NaCl}$ (Figure $2 \mathbf{H}$ ). The growth rate of the bacteria varied between species when the temperature was increased. All the species reached stationary phase after $36-48 \mathrm{~h}$, at $37^{\circ} \mathrm{C}$ and after $42-60 \mathrm{~h}$, at $42^{\circ} \mathrm{C}$ (Figures 1B,C). Rather than considering all 63 different species of Bifidobacterium, we selected species representative of the groups of Bottacini et al. (2014): B. animalis (DSM 10140), B. adolescentis (DSM 20083), and B. longum (DSM
20219) (Bottacini et al., 2014). We subjected them to different conditions, and observed any morphological changes with SEM. Figures 2, 10 clearly demonstrates the effects of each condition on the Bifidobacterial morphology. Bifidobacterium adolescentis (DSM 20083) metamorphosed from a bifid to a rod when grown on medium supplemented with alanine (A), asparagine $(\mathrm{N})$, or $0.25 \mathrm{M} \mathrm{NaCl}$. When B. longum (DSM 20219) was grown on medium supplemented with the four amino acids (Ala, Asp, Glu, and Ser), or separately with alanine (A) or asparagine $(\mathrm{N})$, its morphology changed from elongated rods to bifids. However, B. animalis. subsp. lactis (DSM 10140) remained in the rod form, irrespective of its growth conditions.

\section{Supplementary Data to Understand the Other Bifidobacterial Species}

Another five species, B. thermacidophilum (DSM 15837), B. infantis (DSM 20088), B. asteroides (DSM 20089), B. animalis (DSM 20105), and B. breve (DSM 20213) was also subjected to the different conditions described above, and the results are shown in the Supplementary Figure S1. We noted a marked change in the morphology of $B$. thermacidophilum from rod- to bifidshaped when grown on medium containing tryptophan. When $B$. asteroides was grown at $37^{\circ} \mathrm{C}$, a mixed population of rodand bifid-shaped bacteria were observed. Morphological changes were also observed when $B$. breve was grown with all four amino acids or with alanine alone, when it metamorphosed from rod- to 
bifid-shaped cells. Bifidobacterium infantis changed from rods to coccoids at $42^{\circ} \mathrm{C}$. Almost all the other strains of Bifidobacterium maintained the rod shape under all the conditions tested, except as noted above. These findings confirm that B. animalis maintains its rod form irrespective of the conditions, implying that this morphology is an intrinsic characteristic. Therefore, B. animalis may be used as a reliable control when investigating the bifid nature of $B$. adolescentis (in MRS, at $37^{\circ} \mathrm{C}$, and under anaerobic conditions). B. adolescentis and B. animalis (a control for rod shape) were exposed to other variable conditions, including various $\mathrm{pH}$ (Figure 3), for different growth periods (Figure 4), and in different concentrations of salt (Figure 5). The bifid shape was maintained by $B$. adolescentis under all the conditions tested, whereas the control, B. animalis, retained the rod form. The bifid shape of $B$. adolescentis were also retained when it was grown for three successive generations under normal conditions (Figure 6), in all of which the bifid shape was retained. This further affirms that the intrinsic bifid nature in the case of $B$. adolescentis, and rod shape in the case of B. animalis.

We also found that most Bifidobacterium species stably maintained their morphological (rod) shape, irrespective of the conditions. As shown in the Figure 2, all the Bifidobacterium species were intrinsically rod-shaped, except $B$. adolescentis. Few of them adopted the bifid structure, and only under stress conditions (Figures 2C,D,F,G B. longum; where C, D conditions corresponds to $B$. breve [shown in the Supplementary Information], E condition corresponds to $B$. thermacidophilum [shown in the Supplementary Information], and D, F, G conditions corresponds to $B$. adolescentis) [where $C$ refers to the combination of four amino acids; $\mathrm{D}$ refer to alanine; $\mathrm{E}$ refers to tryptophan; F refers to asparagine; $G$ refers to $0.2 \mathrm{M}$ sodium acetate). However, $B$. adolescentis is intrinsically bifid-shaped and maintains its bifid morphology irrespective of the ambient conditions.

\section{Caenorhabditis elegans as a Model Organism for Pleomorphism Studies}

To understand the bifid form of $B$. adolescentis and the forms of other Bifidobacterium, C. elegans was fed with different Bifidobacterium species and observed microscopically and by SEM. Based on chemotaxis study B. adolescentis and B. longum was considered for the study. Only $B$. adolescentis maintained the bifid shape in the gut of $C$. elegans. The B. adolescentis-fed C. elegans were dissected and centrifuged, and the supernatant was spread on an MRS agar plate. The numbers of bacteria were counted. The bacterial genomic DNA was isolated, and the $16 \mathrm{~S}$ rRNA was amplified and analyzed, confirming that the isolated bacteria were $B$. adolescentis. The same culture was also subjected to an F6PPK assay, which confirmed the bacterial identity. The same bacteria were examined with confocal microscopy (Figure 7), which confirmed their bifid morphology. Bifidobacteria species other than B. adolescentis maintained their intrinsic rod shape in the digestive tracts of the worms, confirming that this is their intrinsic shape, and changes only under stress conditions.
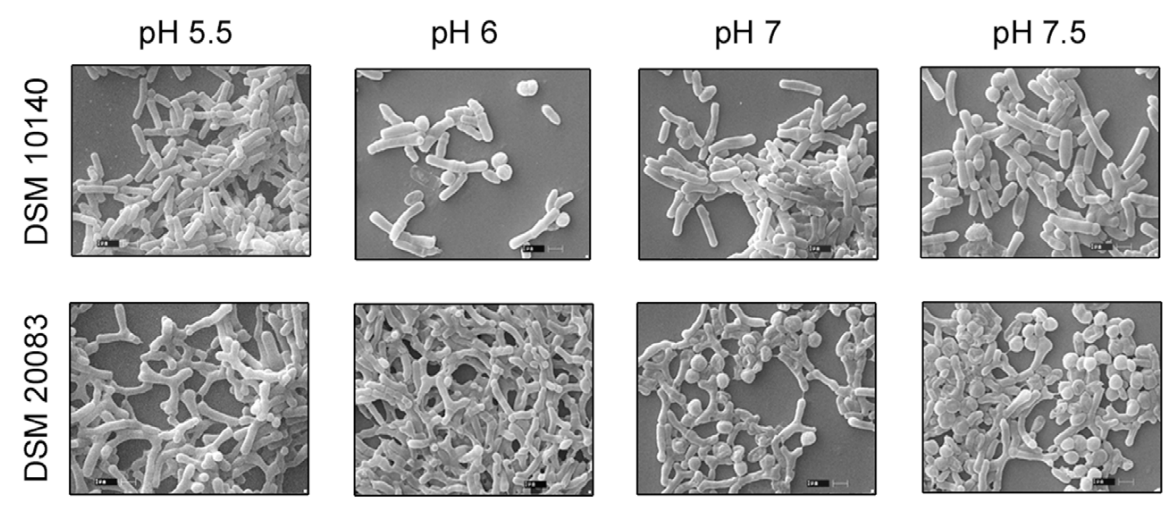

FIGURE 3 | Scanning electron microscopy of strains DSM 10140 (B. animalis subspp. lactis), DSM 20083 (B. adolescentis) in different pH condition.
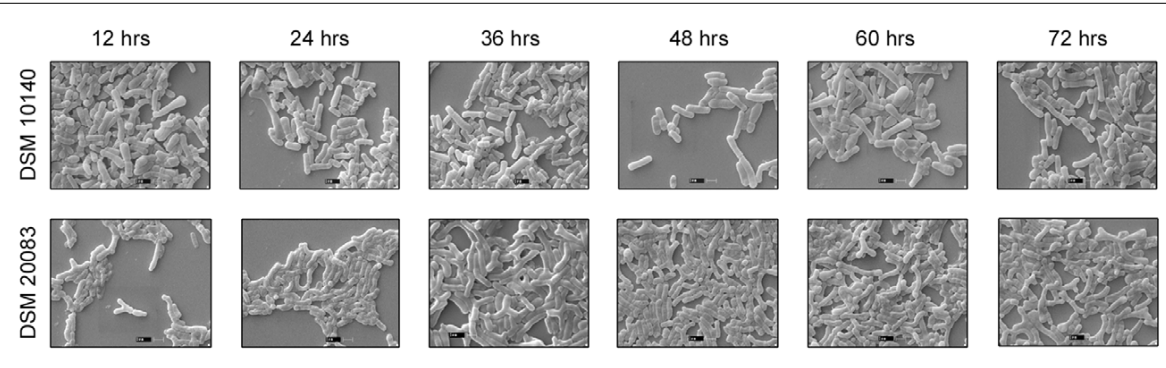

FIGURE 4 | Scanning electron microscopy of strains DSM 10140 (B. animalis subspp. lactis), DSM 20083 (B. adolescentis) in different time interval. 


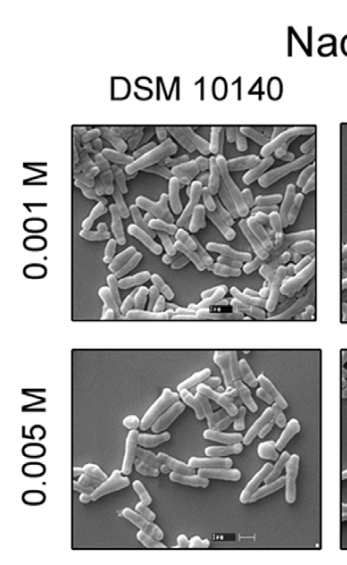

DSM 20083
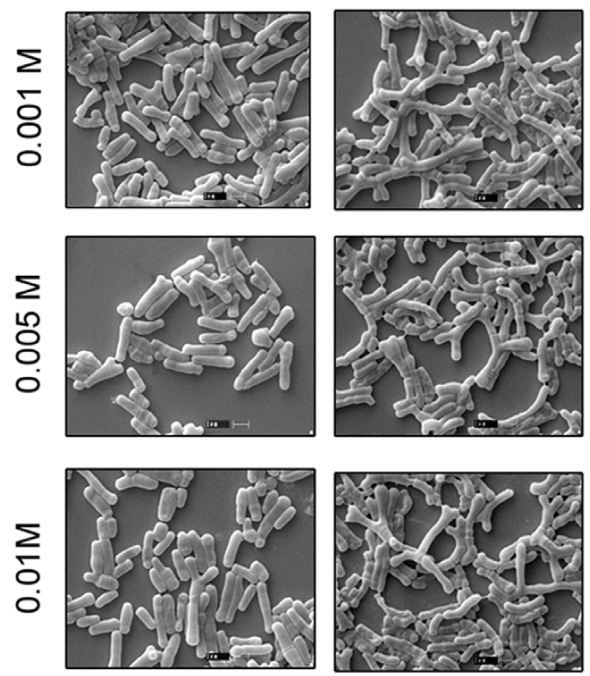
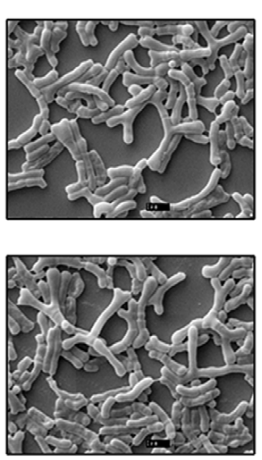

\section{$\mathrm{Cacl}_{2}$}

DSM 10140
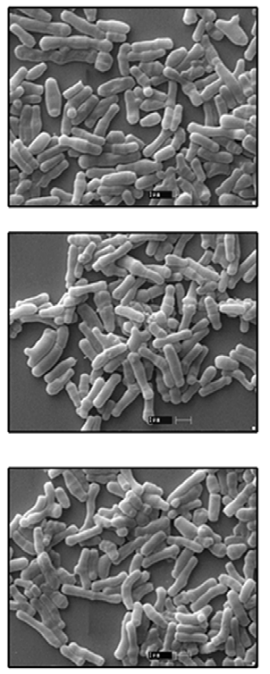

FIGURE 5 | Scanning electron microscopy of strain DSM 10140 (B. animalis subspp. lactis), and DSM 20083 (B. adolescentis) in different salts with varied concentration.

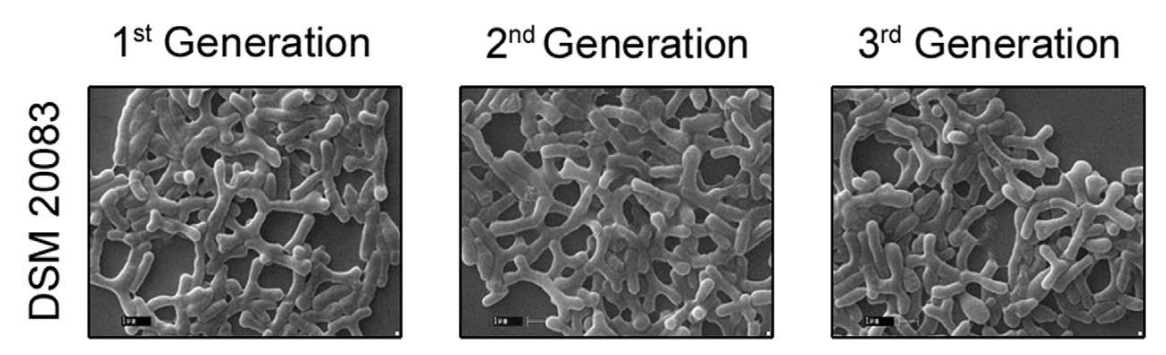

FIGURE 6 | Scanning electron microscopy of strain DSM 20083 (B. adolescentis) at three different generations.
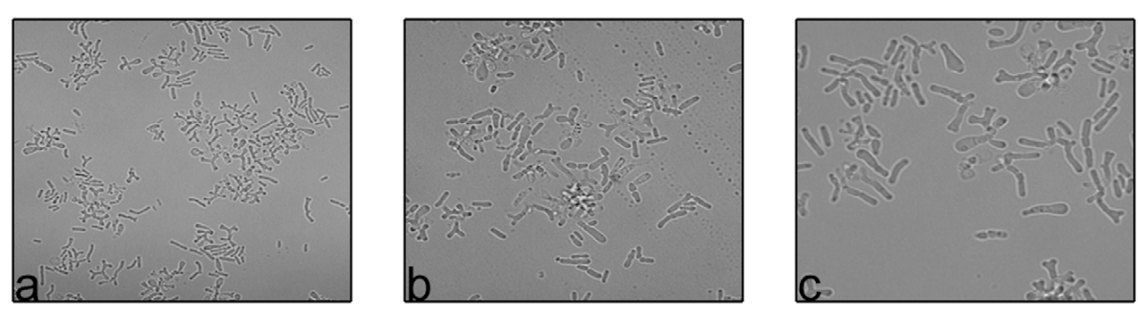

FIGURE 7 | Confocal images of $\boldsymbol{B}$. adolescentis at different magnification (a) Mag- 10x, (b,c) Mag- 60x.

\section{Bifidobacterium is the Preferred Food of C. elegans}

Experiments were performed to understand the ability of C. elegans to consume Bifidobacterium. The food chosen by C. elegans from among probiotic organisms and a control was estimated both qualitatively (Figures $\mathbf{8 A}-\mathbf{D}$ ) and quantitatively (Figure 8E), and represented as the CI. The CI for B. longum was 8.5 and 9.8 at 12 and $24 \mathrm{~h}$, respectively, and was
0.7 for B. adolescentis at $12 \mathrm{~h}$. C. elegans contained no $B$. animalis or $B$. adolescentis at $24 \mathrm{~h}$, indicating that $C$. elegans had not selected them. To validate the bacterial morphology in vivo, nematodes that had consumed B. longum or $B$. adolescentis were fixed and examined with SEM to determine the shapes of the bacteria at different magnifications (Figure 9).

The results in the Figure $\mathbf{8 E}$ clearly show that the nematodes greatly preferred Bifidobacterium to E. coli OP50 as its staple 

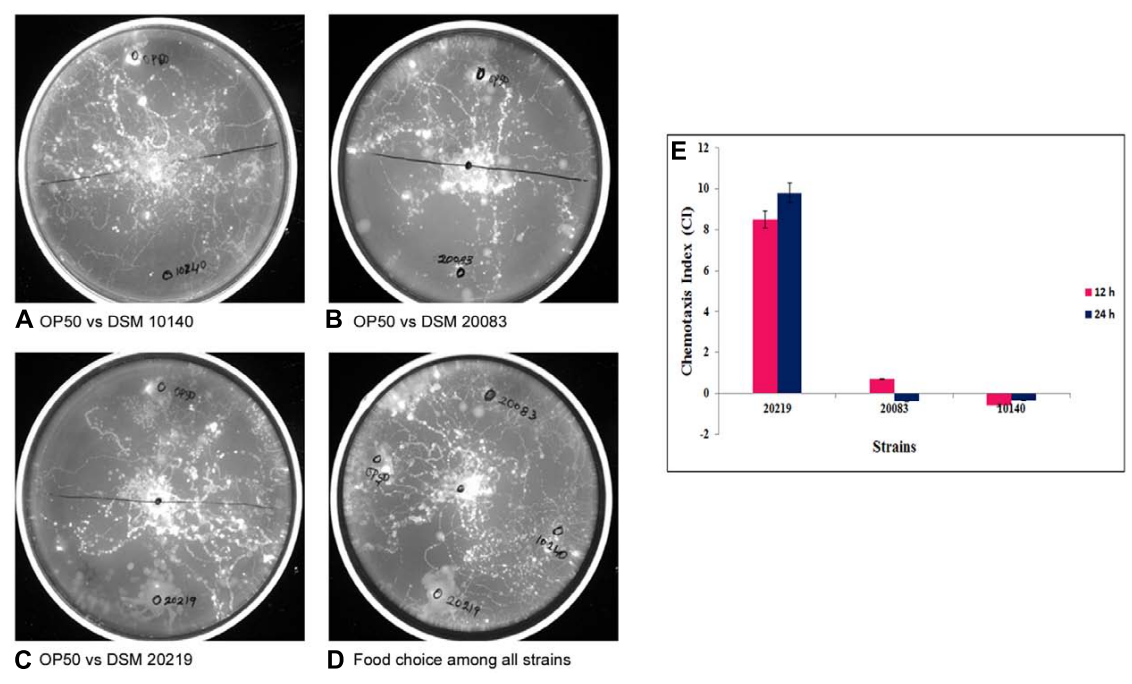

FIGURE 8 | Chemotaxis assay and chemotactic index $(\mathrm{Cl})$ for understanding the food of choice among the other strains by the Caenorhabditis elegans in comparison with control Escherichia coli OP50 vs. (A) DSM 10140 (B) DSM 20083 (C) DSM 20219 and (D) in combination with all. (E) If Cl = -1.0 represents complete preference for E. coli OP50. +1.0 represents complete preference for the test bacterium. 0.0 represents an equal distribution.

food. Caenorhabditis also expressed a preference among the three different Bifidobacterium species tested, so C. elegans may be the best model organism in which to investigate this polymorphism of Bifidobacterium. We assayed the preference of $C$. elegans for bacterial species $B$. longum, B. adolescentis, and B. animalis. The nematodes showed a preference for B. longum over B. adolescentis and $B$. animalis (Figures $\mathbf{8 A - D}$ ). The histogram shows the CIs of wild-type C. elegans for bacterial strains B. longum, $B$. adolescentis, and B. animalis at 12 and $24 \mathrm{~h}$ (Figure 8E). The data are the means \pm SD of three independent biological replicates.

The health benefits of probiotic microbes are only observed when they reach the colon, after passing through the small intestine (low $\mathrm{pH}$ ), and large intestine (alkaline conditions). During their progress, they are exposed to very harsh conditions. To understand the effects on their morphology, these Bifidobacteria were subjected to different $\mathrm{pH}$. When exposed to increasing $\mathrm{pH}$ (Figure 3), B. adolescentis slowly became coccoid, with a dramatic reduction in bifid bacteria. At $\mathrm{pH} 5.5$, they maintained the bifid structure, but as the $\mathrm{pH}$ increased to 6 , the bifid structure started to diminish, and at $\mathrm{pH} 7$ and 7.5, the number of coccoid structures predominated, although no pointed elongated rods were observed (Figure 3). This phenomenon indicates that before it reaches its destination, the morphology of $B$. adolescentis changes frequently to accommodate the ambient conditions. Bacterial movement may be faster in the coccoid state, and this state may be favored when no adhesion is required. For adhesion, a larger and rougher bacterial surface area is required, and therefore a bifid shape is favored. Thus, B. adolescentis always maintained its bifid shape, unless it senses adverse conditions, when its morphology changes.

When Bifidobacterial species were grown in the presence of different salts ( $0.2 \mathrm{M}$ sodium acetate or $0.25 \mathrm{M}$ sodium chloride)
(Kojima et al., 1968), SEM clearly showed that there were no dramatic changes in their morphologies (Figures 2G,H). A slight elongation of the rods was the only visible difference between salt-exposed $B$. longum and control B. longum. Importantly, the presence of lower concentration (0.001-0.01 M) $\mathrm{NaCl}$ did not affect the morphology of $B$. adolescentis, and its bifid structure did not change to the rod form. The bifid structure was observed at lower concentrations of salt, and as the concentration of salt increased, the bacteria assumed the rod shape. The rod structures started to diminish at a salt concentration of 0.005$0.01 \mathrm{M}$, whereas the bifid form started to shorten, until at $0.25 \mathrm{M}$ salt, no bifid structures were seen (Figure $\mathbf{2 H}$, condition of B. adolescentis).

Scanning electron microscopy also showed that Bifidobacterium other than $B$. adolescentis adopted the bifid form, but only very rarely, and only under stress conditions (Figures 2B-H). Therefore, it is clear that the bifid form is an intrinsic to $B$. adolescentis, but not to the other Bifidobacterium species, although a few Bifidobacterial species adopt the bifid form under stress conditions. Kojima et al. (1968) reported that changes to the bifid form occurred in different generations of Bifidobacterium, but our study clearly shows that the first, second and third generations of $B$. adolescentis maintained the bifid morphology (Figure 6). Dramatic changes in B. adolescentis were only observed when it was exposed to different $\mathrm{pH}(\mathrm{pH}$ 5.5-7.5), when it adopted a coccoid shape (Figure 3). Therefore, the number of coccus-shaped bacteria was much higher depicted in the initiation of coccus formation, but reverted back to the bifid form. $\mathrm{CaCl}_{2}$ plays a significant role in the conversion of Bifidobacterium from the bifid shape to the rod form, suggesting the involvement of calmodulin or a similar molecule. This phenomenon was observed when $B$. adolescentis were subjected to different concentrations of $\mathrm{CaCl}_{2}(0.001-0.01 \mathrm{M})$. As the calcium concentration increased, the cells became rod-shaped, 


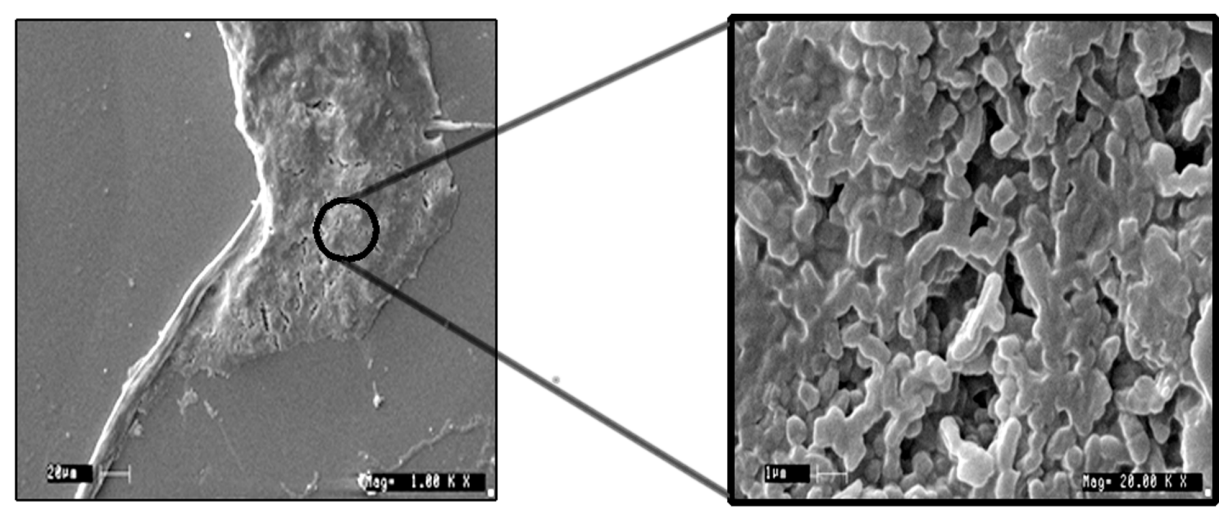

A DSM 20083(B.adolescentis) in C.elegans

B DSM 20083(B.adolescentis) in C.elegans

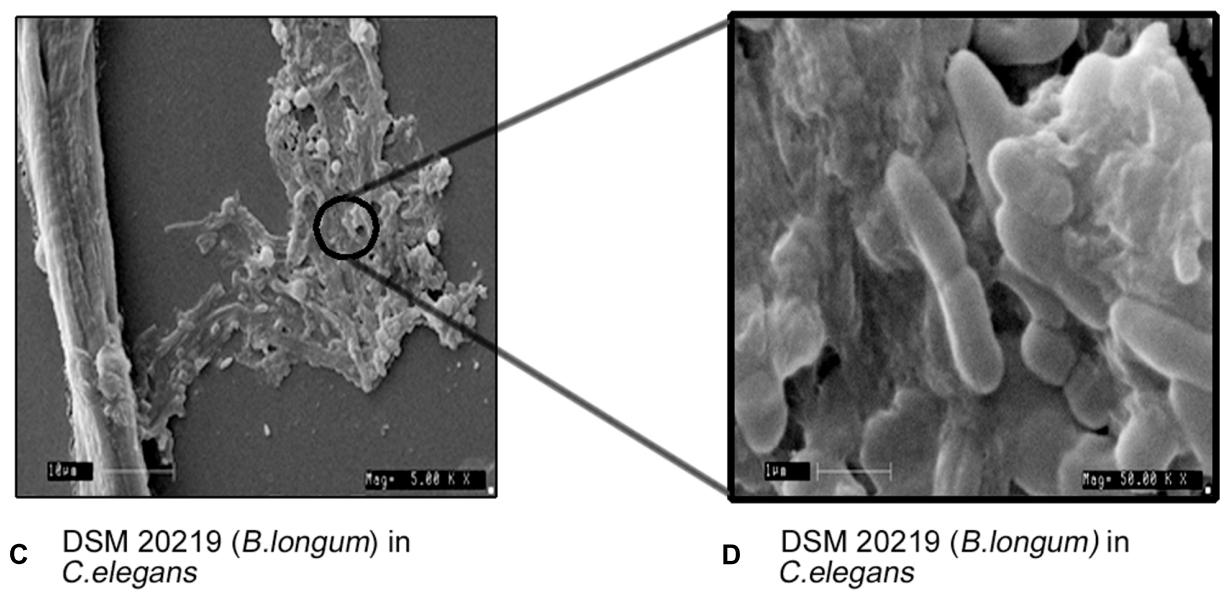

FIGURE 9 | Morphology of B. adolescentis (DSM 20083) and, B. longum (DSM 20219) in C. elegans with different magnification (Mag). (A) DSM 20083 (B. adolescentis) in C. elegans Mag-1000x; (B) DSM 20083 (B. adolescentis) in C. elegans Mag-20,000x; (C) DSM 20219 (B. longum) in C. elegans Mag-5000x; (D) DSM 20219 (B. longum) in C. elegans Mag-50, 000x.

short, and bulging, with terminal buttons, and they also aggregated (Figure 5).

We examined B. longum in experiments similar to those described above because it bears an intrinsic elongated rod structure. As recorded in Figure 2, no dramatic changes were observed under different conditions, except when the cells were exposed to $42^{\circ} \mathrm{C}$, their rod structures changed to extend elongated rods. At higher oxygen concentrations (10\%), they aggregated and became elongated, with button/hyphal structures at their ends. When the concentration of oxygen was reduced, the hypha-like structures and cell aggregation, decreased (data not shown). A low concentration of calcium chloride $(0.001 \mathrm{M})$ caused some defects, including cell shrinkage, the formation of wrinkles or holes, etc. As the levels increased from 0.005 to $0.01 \mathrm{M}$, the cells started out to change from rod-shaped to extensively elongated rods, as well as the bifid form. At all concentrations of $\mathrm{CaCl}_{2}$, the ratio of bifid-shaped B. adolescentis were drastically reduced in number (Figure 5, DSM 20083). Structural alterations of different Bifidobacterial species subjected to different conditions are summarized in Figure 10.

\section{DISCUSSION}

Many researchers have claimed that the Bifidobacterium species are strictly anaerobic, but no research has supported this theory. From our findings, we conclude that the Bifidobacterium species are microaerophilic because they can grow in the presence of a certain level of oxygen after the first generation. Weiss (1933) and Weiss and Rettger (1938) reported that L. bifidus and L. acidophilus is closely related, and are variants of the same species. This hypothesis was rejected for lack of evidence. Subsequently, Orla-Jensen (1943) reported that B. bifidum should be included in the order Actinomycetales. Weiss and Rettger later concluded that respect were two different kinds of Bifidobacterium: type 1 is aerobic and unbranched during primary isolation, and type 2 is anaerobic and branched. Weiss and Rettger (1938) proposed that all these organisms should be retained in Lactobacillus, but not in Actinomycetales. Established on the hypothesis of Weiss and Rettger, the Topley and Wilson's Microbiology and Microbial Infection and Bergey's Manual of Systematic Bacteriology adopted this classification. Orla-Jensen then proposed that the genus name should be converted to 


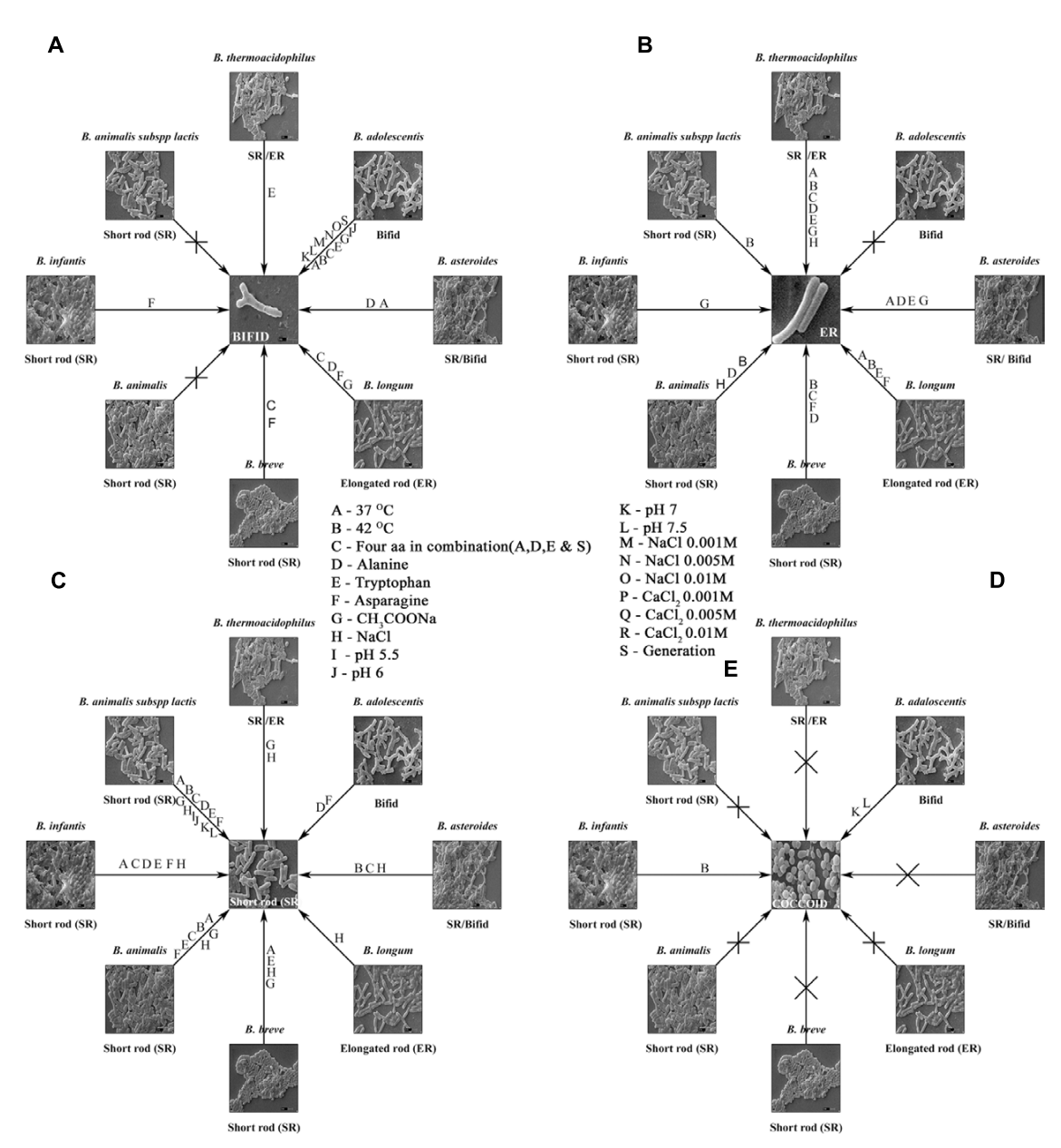

FIGURE 10 | Bifidobacterial species subjected to different conditions and their response are shown in the form of a wheel. (A-E) Shows summary of the Bifidobacteria and their morphologies after exposure to different conditions. Different strains of Bifidobacteria and their inter changed morphologies after subjected to different conditions are depicted. (A) bifid shape, (B) elongated rod (ER), (C) short rod (SR), (D) cocci, (E) different conditions.

'Corynebacterium.' It was reasoned that the use of different growth media for these bacteria may have caused the confusion concerning their nomenclature. These matters remain open today because most of these species are nevertheless brought up to as Bifidobacterium, although most are rod-formed. In 1967, using simple microscopy (not SEM), Masami Kojima found that cations play a significant role in the initiation of the bifid form. Nevertheless, we found that cations do not play a substantial role in bifid formation, although they induce the formation of elongated rods. To see the effects of $\mathrm{NaCl}$ on the bifidobacterial bifid and rod shapes, B. animalis subsp. lactis and $B$. adolescentis were grown in media containing 0.001-0.01 M salt (Figure 5) (Kojima et al., 1968). Both strains maintained their intrinsic morphologies, although at $0.25 \mathrm{M} \mathrm{NaCl}$, B. adolescentis reverted from a bifid to a rodshaped structure (Figure $\mathbf{2 H}$ ). The B. adolescentis changes its intrinsic nature of bifid to rod when they are grown in bifid media (Supplementary Figure S2) and remains same in the presence of amino acid such as asparagine, tryptophan, alanine (individually) (Supplementary Figure S3) which is necessary for the branching of the bacteria (Husain et al., 1972).

These present studies showed that $\mathrm{CaCl}_{2}$ plays a major part in abolishing the bifid shape, which is the intrinsic morphology of $B$. adolescentis. Nevertheless, no significant conclusions were drawn from Kojima et al. (1970) research group involving the effects of cations on the bifid form of Bifidobacterium. Kojima et al. (1968) concluded that a calciumfree environment induces the bifid state, whereas the presence of calcium induces the bacilloid form. Yet, in the present study, $\mathrm{CaCl}_{2}$ induced the bacillus form of $B$. adolsecentis, despite its intrinsic bifid morphology. Therefore, although the bifid form is the intrinsic morphology of a few Bifidobacterial species, this is not absolute. $\mathrm{CaCl}_{2}$ may be considered a suppressor of the bifid state, supporting the proposition that there are calmodulins, calmodulin-like proteins in the Bifidobacterium species.

Our SEM analysis clearly shows that the growth medium (bifidobacteria broth or MRS) and different conditions (such as 
various temperatures, $\mathrm{pH}$, and salts) do play a major part in the pleomorphic morphology of the Bifidobacterium. The presence and absence of amino acids did not (dramatically) affect their morphologies, converting the rod structure to the bifid form or to cocci, or the bifid form to the rod form. Not all Bifidobacterium have a bifid morphology, and only a very few are intrinsically bifid-shaped, such as B. adolescentis. It is well-demonstrated in this study that the intrinsic morphologies of these bacteria are rod-shaped, and all but a few do not adopt the bifid form, even after exposure to stress conditions. Thus, the rod structure is the intrinsic morphology of most bifidobacterial species. These data confirm the theory that the name 'Bifidobacterium' is a misnomer. Therefore, taxonomists must revisit this name by picking up more data in the near future.

As we expected, Bifidobacterium was the intellectual nourishment of choice for the eukaryotic model system, C. elegans, although the standard food offered C. elegans experimentally has been E. coli OP50. The nematodes tended to indicate a preference for Bifidobacterium over E. coli OP50. C. elegans also differentiates among the Bifidobacterium, as seen in Figure 8. Of the three Bifidobacterium species given as food, C. elegans preferred B. longum over B. adolescentis and $B$. animalis. The morphology of Bifidobacteria is intrinsically determined; the external factors don't have anything to do with the same, example $B$. adolescentis. Equally it is the only strain out of eight tested in our study retained its bifid shape in the extremities.

\section{AUTHOR CONTRIBUTIONS}

D executed the idea; RK conceptualized, planned, designed, analyzed, Interpreted results and wrote the manuscript. SR and BK all the C. elegans work planning and execution.

\section{REFERENCES}

Beerens, H., Gavini, E., and Neet, C. (2000). Effect of exposure to air on 84 different strains of Bifidobacteria. Anaerobe 6, 65-67. doi: 10.1006/anae.1999.0317

Blaurock, G. (1937). Zur Physiologie der Bifidusbakterien. Monatsschr. Kinderheilk. 68, 304-309.

Bliuhdorn, K. (1910). Untersuchugen uiber den Bacillus bifidus communis und den so gen. Bacillus acidophilus (Streptobacillus faecalis). Jahrb. Kinderheilk 72, 693-704.

Bottacini, F., Ventura, M., van Sinderen, D., and Motherway, M. O. C. (2014). Diversity, ecology and intestinal function of bifidobacteria. Microb. Cell Fact. 13(Suppl. 1), S4. doi: 10.1186/1475-2859-13-S1-S4

Boventer, K. (1938). Untersuchungen fiber das bacterium bifidum. Zentralbl. Bakteriol. Parasitenk. Infektionskr. Hyg. Abt. Orig. 142, 419-430.

Brenner, S. (1974). The genetics of Caenorhabditis elegans. Genetics 77, 71-94.

Cruickshank, R. (1925). Bacillus bifidus: its characters and isolation from the intestine of infants. J. Hyg. 24, 241-254. doi: 10.1017/S0022172400008718

Dong, X., Xin, Y., Jian, W., Liu, X., and Ling, D. (2000). Bifidobacterium thermacidophilum sp. nov., isolated from an anaerobic digester. Int. J. Syst. Evol. Microbiol. 50, 119-125. doi: 10.1099/00207713-50-1-119

Garsin, D. A., Sifri, C. D., Mylonakis, E., Qin, X., Singh, K. V., Murray, B. E., et al. (2001). A simple model host for identifying gram-positive virulence factors. Proc. Natl. Acad. Sci. U.S.A. 98, 10892-10897. doi: 10.1073/pnas.191 378698

\section{ACKNOWLEDGMENTS}

The Department of Biotechnology, India, the Director CFTRI, Mysore are appreciated for providing the facilities and funds. We also acknowledge Mr. Anbalagan, Department of CIFS, SEM and Confocal expert for helping us to capture the best possible pictures. The UGC-CSIR fellowship for D has been highly appreciated.

\section{SUPPLEMENTARY MATERIAL}

The Supplementary Material for this article can be found online at: http://journal.frontiersin.org/article/10.3389/fmicb. 2017.00478/full\#supplementary-material

FIGURE S1 | Scanning electron microscopy of strains DSM 15837 (B. thermoacidophilus), DSM 20088 (B. infantis), DSM 20089 (B. asteroides), DSM 20105 (B. animalis), DSM 20213 (B. breve) with different conditions like (A) $37^{\circ} \mathrm{C}$, (B) $42^{\circ} \mathrm{C}$, (C) media with alanine, aspartate, glutamate, serine, (D) media with alanine, (E) media with tryptophan, (F) media with asparagine, media with $0.2 \mathrm{M}$ sodium acetate, media with $0.25 \mathrm{M}$ sodium chloride, (G) media with $0.2 \mathrm{M}$ sodium acetate, $(\mathbf{H})$ media with $0.25 \mathrm{M}$ sodium chloride.

FIGURE S2 | Scanning electron microscopy of strains DSM 10140 (B. animalis subspp. lactis), DSM 20083 (B. adolescentis) in bifid media at time interval.

FIGURE S3 | Scanning electron microscopy of strains DSM 10140 (B. animalis subspp. lactis), DSM 20083 (B. adolescentis) in bifid media with (A) asparagine, (B) tryptophan, (C) alanine. Food choice assay: assaying the preference of $C$. elegans for bacterial species B. longum, B. adolescentis, and $B$. animalis. Nematodes showed a greater preference for $B$. longum than for B. adolescentis, or B. animalis. Chemotaxis assay: the histogram shows the chemotaxis index $(\mathrm{Cl})$ of wild-type $C$. elegans toward bacterial strains 20219 , 20083, and 10140 after 12 and 24 h. Data are the means \pm SD of three independent biological replicates.

Herter, C. A. (1907). The Common Bacterial Infections of the Digestive Tract and the Intoxications Arising from Them. London: Macmillan. doi: 10.5962/bhl.title. 30633

Homma, N., Ota, J., and Arai, S. (1960). Various carbonates affecting the branching of Lactobacillus bifidus. Jpn. J. Pediatr. 13:1274.

Hope, I. A. (ed.) (1999). C. elegans: A Practical Approach (Vol. 213). Oxford: Oxford University Press.

Husain, I., Poupard, J. A., and Norris, R. F. (1972). Influence of nutrition on the morphology of a strain of Bifidobacterium bifidum. J. Bacteriol. 111, 841-844.

Kawasaki, S., Mimura, T., Satoh, T., Takeda, K., and Nimura, Y. (2006). Response of the microaerophilic Bifidobacteria spp B. boum and B. thermophilum to oxygen. Appl. Environ. Microbiol. 72, 6854-6858. doi: 10.1128/AEM. 01216-06

Kendall, A. I., and Haner, R. C. (1924). Bacillus Bifidus: LXXI. studies in bacterial metabolism. J. Infect. Dis. 35, 77-88. doi: 10.1093/infdis/35.1.77

Kojima, M., Suda, S., Hotta, S., and Hamada, K. (1968). Induction of pleomorphism in Lactobacillus bifidus. J. Bacteriol. 95, 710-711.

Kojima, M., Suda, S., Hotta, S., and Hamada, K. (1970). Induction of pleomorphy and calcium ion deficiency in Lactobacillus bifidus. J. Bacteriol. 102, 217-220.

Meile, L., Ludwig, W., Rueger, U., Gut, C., Kaufmann, P., Dasen, G., et al. (1997). Bifidobacterium lactis sp. nov., a moderately oxygen tolerant species isolated from fermented milk. Syst. Appl. Microbiol. 20, 57-64. doi: 10.1016/S07232020(97)80048-3

Mitsuoka, T. (1969). Comparative studies on bifidobacteria isolated from the alimentary tract of man and animals (including descriptions of Bifidobacterium 
thermophilum nov. spec. and Bifidobacterium pseudolongum nov. spec). Zentralbl. Bakteriol. Orig. 210, 52-64.

Moro, E. (1905). Morphologische und biologische Untersuchungen über die Darmbakterien des Säuglings. Jb. Kinderheik 62:687.

Norris, R. F., Flanders, T., Tomarelli, R. M., and György, P. (1950). The isolation and cultivation of Lactobacillus bifidus: a comparison of branched and unbranched strains. J. Bacteriol. 60, 681-696.

Orla-Jensen, S. (1943). The lactic acid bacteria. Ergainzungsband. Mem. Acad. Roy. Sci., Danemark, Sect. Sci. Biol. 2, 1-145.

Reuter (1963). Identisch mit Bifidobacterium adolescentis. Parasitenkd. Infektionskr. Hyg. Abt. 1 Orig. Reihe A 226, 257-263.

Rodella, A. (1908). Magen Carcinoma und Milchsaure Bacillen. Centralbl. f. Bakt., Abt. I 47, 445-466.

Roos, C. (1927). Cultural characteristics (variation of Bacillus acidophilus). J. Lab. Clin. Med. 12:1053.

Ruiz, L., Gueimonde, M., Ruas-Madiedo, P., Ribbera, A., Delos ReyesGavilan, C., Ventura, M., et al. (2011). Molecular clues to understand the aerotolerance phenotype of Bifidobacterium animalis subsp. lactis. Appl. Environ. Microbiol. 78, 644-656. doi: 10.1128/AEM. 05455-11

Scardovi, V., and Trovatelli, L. D. (1969). New species of bifid bacteria from Apis mellifica L. and Apis indica F. A contribution to the taxonomy and biochemistry of the genus Bifidobacterium. Zentralbl. Bakteriol. Parasitenkd. Infektionskr. Hyg. 123, 64-88.

Stiernagle, T. (1999). "Maintenance of C. elegans," in C. elegans A Practical Approached, ed. I. A. Hope (Oxford: Oxford University Press), 51-67.

Sundman, V., Björksten, K. A., and Gyllenberg, H. G. (1959). Morphology of the bifid bacteria (organisms previously incorrectly designated Lactobacillus bifidus) and some related genera. Microbiology 21, 371-384.

Synder, M. L., Slawson, M. S., Bullock, W., and Parker, R. B. (1967). Studies on oral filamentous bacteria. II. Serological relationships within the genera Actinomyces, Nocardia, Bacterionema and Leptotrichia. J. Infect. Dis. 117, 341-345. doi: 10.1093/infdis/117.4.341
Tissier, H. (1900). Recherches sur la Flore Intestinale des Nourrissons: état Normal et Pathologique. Paris: G. Carré et C. Naud.

Tissier, H. (1905). Repartition des microbes dans l'intestin du nourisson. Ann. Inst. Pasteur 19, 109-123.

Tissier, M. H. (1899). La reaction chromophile d'Escherich et le Bacterium coli. CR Acad. Sci. Ser. III Sci. Life Sci. 51, 943-945.

Tomarelli, R. M., Norris, R. F., and Gyorgy, P. (1949a). Inability of vitamin B12 to replace the deoxyriboside requirement of a Lactobacillus bifidus. J. Biol. Chem. $179,485-486$.

Tomarelli, R. M., Norris, R. F., Gyorgy, P., Hassinen, J. B., and Bernhart, F. W. (1949b). The nutrition of variants of Lactobacillus bifidus. J. Biol. Chem. 181, $879-888$.

Ward, S. (1973). Chemotaxis by the nematode Caenorhabditis elegans: identification of attractants and analysis of the response by use of mutants. Proc. Natl. Acad. Sci. U.S.A. 70, 817-821. doi: 10.1073/pnas.70.3.817

Weiss, J. E. (1933). Lactobacillus bifidus Tissier and Its Biological Position in the Group of Aciduric Organisms. Ph.D. Dissertation, Yale University, New Haven, CT.

Weiss, J. E., and Rettger, L. F. (1938). Taxonomic relationships of Lactobacillus bifidus (B. bifidus Tissier) and Bacteroides bifidus. J. Infect. Dis. 62, 115-120. doi: $10.1093 /$ infdis/62.1.115

Conflict of Interest Statement: The authors declare that the research was conducted in the absence of any commercial or financial relationships that could be construed as a potential conflict of interest.

Copyright (c) 2017 Dhanashree, Rajashekharan, Krishnaswamy and Kammara. This is an open-access article distributed under the terms of the Creative Commons Attribution License (CC BY). The use, distribution or reproduction in other forums is permitted, provided the original author(s) or licensor are credited and that the original publication in this journal is cited, in accordance with accepted academic practice. No use, distribution or reproduction is permitted which does not comply with these terms. 